\title{
FIRST EXPERIMENTALLY OBSERVED SELF-ORGANIZED PARAMETRIC DOWN CONVERSION IN OPTICAL FIBERS
}

\author{
P. Chmela, O. Samek and M. Minarčík \\ Institute of Physical Engineering, Brno University of Technology \\ Technická 2, 61669 Brno, Czech Republic
}

\begin{abstract}
The theoretically predicted self-organized parametric down conversion (the amplification of fundamental radiation on account of the energy of second-harmonic radiation) in doped optical fibers was experimentally demonstrated for the first time and a weak permanent second-order susceptibility grating in the conditioned fiber was identified.
\end{abstract}

PACS numbers: 42.65.Ky, 42.81.Qb

Since the discovery of self-organized frequency doubling in germanium-phosphorous-doped optical fibers by Österberg and Margulis [1] many ingenious experiments were performed and brilliant theoretical models were proposed (for review, see Refs. [2-6]), however, the phenomenon remains a puzzle hitherto.

The first puzzling question was how the initial second-harmonic (SH) radiation can be generated in a centrosymmetric optical fiber by the fundamental pump alone, without any external SH seed. Most physicists have believed that the initial self-seeded second-harmonic generation (SHG) occurs due to the quadrupole interactions [7]. However, other models have been suggested as well. A SHG from quantum noise owing to the fifth-order nonlinearity has been considered to explain the initial rise of self-seeded SHG [8]. Another model considering an exotic four-wave mixing with the Stokes wave at zero frequency and the anti-Stokes wave at $2 \omega$ was outlined as well [2]. However, none of these models has been definitively accepted and the question of initial self-seeded SHG remains still open.

The essential question is which mechanism can break the symmetric structure of the fiber or fiber preform being conditioned to SHG, and how the proper spatially periodic second-order susceptibility $\left(\chi^{(2)}\right)$ structure can be created that enables an efficient quasi-phase-matched SHG.

It has been ascertained that a strong spatially periodic permanent dc electric field being of the order of some $\mathrm{kV} / \mathrm{cm}$ is created at the interaction of fundamental and $\mathrm{SH}$ radiation in the $\mathrm{SHG}$-conditioned samples [9-11]. This dc electric field deprives the centrosymmetric structure of isotropic medium $[12,13]$ and enables an efficient quasi-phase-matched SHG [14-16]. 
The relevant question occurs what is the physical mechanism being responsible for creating the relatively strong dc electric field in a doped-glass fiber or bulk sample being conditioned to SHG.

The first phenomenological model was outlined by Stolen and Tom [17] who proposed that an internal dc electric field is produced owing to the non-degenerate third-order optical rectification

$$
\begin{aligned}
\boldsymbol{P}_{\mathrm{dc}}^{(3)} & =\frac{1}{2}\left[\chi^{(3) \mathrm{rect}}(\omega+\omega-2 \omega): \boldsymbol{E}_{\omega} \boldsymbol{E}_{\omega} \boldsymbol{E}_{2 \omega}^{*}+\text { c.c. }\right] \\
& =\boldsymbol{e}_{\mathrm{dc}} \chi_{\mathrm{eff}}^{(3) \mathrm{rect}}\left|A_{\omega}\right|^{2}\left|A_{2 \omega}\right| \cos (\Delta k z-\Delta \varphi),
\end{aligned}
$$

where $\boldsymbol{E}_{\omega}=\boldsymbol{e}_{\omega} A_{\omega} \exp \left[\mathrm{i}\left(\omega t-k_{\omega} z\right)\right]$ and $\boldsymbol{E}_{2 \omega}=\boldsymbol{e}_{2 \omega} A_{2 \omega} \exp \left[\mathrm{i}\left(2 \omega t-k_{2 \omega} z\right)\right]$ are the electric intensities of fundamental and SH light waves, $\Delta k=k_{2 \omega}-2 k_{\omega}$ represents the phase-mismatch factor, $\Delta \varphi=\varphi_{2 \omega}-2 \varphi_{\omega}$ is the relative phase shift between the interacting waves and $\chi^{(3) \text { rect }}(\omega+\omega-2 \omega)$ is the third-order rectification susceptibility.

The formation of a system of elementary electric dipoles was assumed and the internal dc electric field created, $\boldsymbol{E}_{\mathrm{dc}}=-\left(\nu / \varepsilon_{0}\right) \boldsymbol{P}_{\mathrm{dc}}^{(3)}(\nu$ being a dimensionless constant and $\varepsilon_{0}$ is the permittivity of vacuum), was believed to modify the centrosymmetric structure of the isotropic medium so that the SHG can occur due to the third-order nonlinear polarization [13]

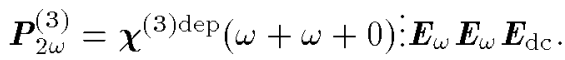

In fact, the Stolen-Tom model describes the self-organized SHG as a fifth-order nonlinear optical interaction that cannot provide any efficient SHG in the instantaneous medium response picture. Even when replacing the rectification susceptibility $\chi^{(3) \text { rect }}$ by a time-dependent coefficient describing the memory effect, the model shows serious difficulties. Though the $\chi^{(2)}$ grating created possesses the right spatial periodicity for an efficient quasi-phase-matched SHG [14-16] the SH radiation produced is shifted by $\pi / 2$ with respect to the $\mathrm{SH}$ seed [5], which is precisely the wrong value to allow a weak SH seeding light to grow in the course of SHG preparation process. In order to remove these difficulties the cumulative third-order optical rectification via a complex response function, being dependent upon the light intensities of writing radiation, was proposed in Ref. [5].

The Stolen-Tom idea [17] gave rise to a group of orientational models that assume the formation of electric-dipole-related asymmetry or microscopic charge separation. The frozen-in periodic dc electric field is believed to be produced by local charge transfer of an electron between two neighbouring defect sites. The macroscopic dc electric-dipole polarization of the area encoded for SHG is then the result of the vector sum of oriented or induced microdipoles.

Another model explaining the $\chi^{(2)}$ grating formation by macroscopic charge separation due to the photovoltaic effect was proposed by Dianov et al. [22-24]. An ionizing interference of two pump photons and one SH photon is assumed to cause the ejection of an electron from existing (photoinduced) defect centre in a preferred direction, which is trapped at the boundary of the illuminated area. The macroscopic charge redistribution induces then the spatially periodic $\chi^{(2)}$ grating. 
A photogalvanic space-oscillating coherent current $j_{\mathrm{ph}}$ is considered, which is a nonlinear function of the applied radiation field [22-24],

$$
j_{\mathrm{ph}}=\beta E_{\omega}^{2} E_{2 \omega}^{*}+\text { c.c. }=|\beta|\left|A_{\omega}\right|^{2}\left|A_{2 \omega}\right| \cos (\Delta k z-\Delta \varphi+\psi),
$$

where $|\beta|$ is the effective value of the coherent-photovoltaic tensor and $\psi$ represents a phase constant shift that should be determined by the microscopic model of photocurrent, properties of the medium and parameters of radiation [25].

As a response to the photoinduced dc electric field a conduction current $j_{\mathrm{ph}}=\sigma E_{\mathrm{dc}}$ occurs that works against the photogalvanic current. The conductivity $\sigma$ was originally assumed to be dependent on pump wavelength and the concentration of dopant defects only [25].

Using the third Maxwell equation and the continuity equation it is easy to derive the equation for the time evolution of second-order susceptibility grating $[22,23,25]$

$$
\frac{\partial \chi^{(2)}}{\partial t}+\frac{\sigma}{\varepsilon} \chi^{(2)}=\alpha A_{\omega}^{2} A_{2 \omega}^{*} \exp [\mathrm{i}(\Delta k z+\psi)]+\text { c.c. },
$$

where $\varepsilon$ is a permittivity and $\alpha=|\beta| \chi^{(3) \text { dep }} / \varepsilon$.

Experiments were performed to decide the adequacy of either model proposed and the macroscopic charge separation was identified in some bulk doped-glass samples being conditioned to $\mathrm{SHG}[25,26]$.

Even when the photovoltaic model can explain many features of the behaviour of self-organized SHG [24] it shows also serious difficulties.

In order to involve the freezing-in of the photoinduced $\chi^{(2)}$ grating it is necessary to assume that the conductivity $\sigma$ drops immediately to zero after switching off the radiation field, which seems to be rather unnatural.

Moreover, Eq. (4) predicts that the saturation second-order susceptibility $\chi_{\text {sat }}^{(2)}$ depends on the instantaneous electric intensities of the saturation radiation field only, meaning that the resulting saturation state does not reflect the previous history of the preparation process, which is in strong disagreement with experimental observations (see, e.g. experiments by Krol et al. [27] and their discussion in [28]). On the contrary, the process of photoinduced self-organized $\chi^{(2)}$ grating formation is very sensitive to the light intensities of the fundamental and the SH input radiation, and the saturation value $\chi_{\text {sat }}^{(2)}$ is the outcome of the whole previous interaction.

A directional photoionization model of self-organized SHG was elaborated by Anderson et al. [29] who considered two-, three- and four-photon ionizing interference to derive a new formula for steady-state dc electric field in the picture of anisotropic excitation process. Unfortunately, this model is mostly omitted in the relevant literature because the attempts of Lavandy et al. [30] to verify the derived formula for resulting dc electric field, being created in the course of self-organized SHG, failed. In our opinion the failing of Lavandy's verification experiment [30] is a consequence of the fact that the self-organized formation of second-order susceptibility is a cumulative process, but in Anderson's et al. picture [29] it is described as a dynamical equilibrium of the charge redistribution owing to the anisotropic multiphoton ionization interference (see, discussion in [31]). 
Though the Anderson formula for steady-state dc electric field, being formed in the course of self-organized SHG, was found to be in strong disagreement with the experimental observations [30,32], the directional photoionization model yields good predictions for the saturation. It predicts for the saturation value of the ratio $I_{2 \omega} / I_{\omega}^{2}$ ( $I_{\omega}$ and $I_{2 \omega}$ being the light intensities of fundamental and SH radiation, respectively) that

$$
\left(\frac{I_{2 \omega}}{I_{\omega}^{2}}\right)_{\text {sat }}=\left(\frac{\mu_{0}}{\varepsilon_{0}}\right)^{1 / 2} \frac{16 n_{2 \omega} e^{2} \kappa}{n_{\omega}^{2} \hbar \omega^{3} m_{e}}\left[10^{-16} \mathrm{~m}^{2} / \mathrm{W}\right],
$$

where $\varepsilon_{0}$ is the electric permittivity and $\mu_{0}$ - magnetic permeability of vacuum in SI units, $n_{\omega}$ and $n_{2 \omega}$ are the refractive indexes of fundamental and SH waves, $e$ and $m_{\mathrm{e}}$ are the elementary charge and mass of the electron, respectively, $\hbar$ is the reduced Planck constant, and $\kappa$ is a factor related to the ionized electron momentum which is less but of the order unity.

In Anderson's et al. picture the $\mathrm{SH}$ radiation itself is a limiting factor that restricts the maximum SH light intensity.

It is interesting to note that the saturation formula similar to Eq. (5) was derived by another purely phenomenological cumulative local-response model [31] as well.

A number of published experiments on self-organized SHG with the self-seeding and the external SH seeding was evaluated in order to verify the predicted saturation of photoinduced SHG by Eq. (5), and it has been found that the experimentally determined value of $\left(I_{2 \omega} / I_{\omega}^{2}\right)_{\text {sat }}$ ranges from about $0.2 \times 10^{-16} \mathrm{~m}^{2} / \mathrm{W}$ to $2 \times 10^{-16} \mathrm{~m}^{2} / \mathrm{W}[33]$. The uncertainty within one order is dominantly caused by the inaccuracy of the experimental data reported.

It seems very probably that the self-saturation of self-organized SHG is the dominant mechanism, behind moving the photoinduced $\chi^{(2)}$ grating backwards (towards the input end of the fiber) if the preparation process runs sufficiently long time, which was observed by Lacerda et al. [34].

Considering the saturation ratio to be $\left(I_{2 \omega} / I_{\omega}^{2}\right)_{\text {sat }} \approx 10^{-16} \mathrm{~m}^{2} / \mathrm{W}$ and taking the extreme values of pump intensities used in real experiments to be of the order of $10^{14} \mathrm{~W} / \mathrm{m}^{2}$ we arrive at the maximum efficiency of photoinduced SHG predicted, $\left(I_{2 \omega}^{\text {output }} / I_{\omega}\right)_{\text {max }} \approx 10^{-2}$, which is in a very good accord with real experiments.

In conclusion, it seems quite evident that the self-saturation, originally predicted by Anderson et al. [29], represents the unavoidable attribute of the photoinduced self-organized SHG and, consequently, the endeavours to reach higher efficiencies of self-organized SHG with commonly used intensities of fundamental radiation are futile.

As the saturation effect was predicted by the local response models [29, 31], it follows that the same saturation SH light intensity can be reached at any even very short interaction length, if the SHG-preparation process runs for a sufficiently long time, which was also experimentally demonstrated by Lacerda et al. [34], who observed that the $\chi^{(2)}$ grating, created by the self-seeding preparation process, moves slowly back towards the input end of the fiber after reaching the saturation regime, whilst the $\mathrm{SHG}$ efficiency remains constant. 
Another important feature of self-organized SHG is the phase behaviour of generated SH light. The mutual phase shift of the SH radiation generated at the photoinduced $\chi^{(2)}$ grating and the $\mathrm{SH}$ seeding (input) radiation, $\Delta \varphi_{2 \omega}=$ $\varphi_{2 \omega}^{\text {gen }}-\varphi_{2 \omega}^{\text {seed }}$, was measured by Margulis et al. [35] for the first time. This measurement yielded the phase shift of about $90^{\circ}$, which is precisely the wrong value to allow a weak SH seeding light to be enhanced in the course of SHG preparation process. However, the SH seeding intensity was relatively strong and approached the saturation value in this case. For somewhat lower SH seeding intensities other values of $\Delta \varphi_{2 \omega}$ were reported as well [36-38].

In 1996 Lambelet and Feinberg [38] performed a unique experiment in which they measured both the power and phase of $\mathrm{SH}$ output radiation emerging from a short germanium-doped glass fiber being conditioned to SHG in the whole course of preparation process. Using these experimental results, we have computed the evolution of $\Delta \varphi_{2 \omega}(t)$ in one-hour preparation time interval [39]. Our computation has shown that $\Delta \varphi_{2 \omega}(t)$ monotonously increased from its initial value $\Delta \varphi_{2 \omega}(0) \approx$ $70^{\circ}$ and approached $\Delta \varphi_{2 \omega}$, sat $\approx 90^{\circ}$ in the saturation state. The uncertainty of the determination of $\Delta \varphi_{2 \omega}(t)$ was estimated to be at about $10 \%$.

Evidently, the mutual phase shift $\Delta \varphi_{2 \omega}=\varphi_{2 \omega}^{\text {gen }}-\varphi_{2 \omega}^{\text {seed }}$ cannot be considered constant but it varies in the course of SHG preparation process. We believe that the initial value of mutual phase shift of SH seed and SH radiation generated $\Delta \varphi_{2 \omega}(0)$ is crucially dependent on the value of the ratio $I_{2 \omega}^{\text {seed }} / I_{\omega}^{2}$, but its saturation value always approaches $90^{\circ}$.

Taking into account both the saturation and the phase behaviour of self-organized SHG, as well as the memory effect, we arrived at the conclusion that it is impossible to describe these phenomena if using simple material constants as it is usually done in phenomenological electromagnetic theory of optical interactions.

We have proposed a new phenomenological model that describes the self-organized $\chi^{(2)}$ grating formation at the interaction of fundamental and SH waves by means of $\chi^{(2)}$ encoding function as follows [40]

$$
\begin{gathered}
\chi^{(2)}(z, t)=-\exp (\mathrm{i} \Delta k z) \int_{0}^{t} F\left[I_{\omega}(z, t-\tau), I_{2 \omega}(z, t-\tau), \tau\right] \\
\times A_{\omega}^{2}(z, t-\tau) A_{2 \omega}^{*}(z, t-\tau) \mathrm{d} \tau+\text { c.c. },
\end{gathered}
$$

where the $\chi^{(2)}$ encoding function $F(z, t-\tau)$ is complex, it is dependent on the instantaneous light intensities $I_{\omega}$ and $I_{2 \omega}$ and it varies in the course of SHG preparation process, as well as along the fiber; $\Delta k=k_{2 \omega}-2 k_{\omega}$ is the phase-mismatch factor including also the Kerr nonlinearities.

In the approximation of nondepleted pump, $A_{\omega}=$ const, the spatial evolution of SH field amplitude can be described by means of a simple first-order differential equation

$$
\frac{\partial A_{2 \omega}(z, t)}{\partial z}=-\mathrm{i}\left(\frac{\mu_{0}}{\varepsilon_{0}}\right)^{1 / 2} \frac{\omega}{n_{2 \omega}} \chi^{(2)}(z, t) A_{\omega}^{2}(z, t) .
$$
in $[40]$.

The solution of Eqs. (6) and (7) together with the discussion was published 
It has been shown that only the imaginary part of $\chi^{(2)}$ encoding function, $\operatorname{Im}[F(z, t-\tau)]$, contributes effectively to the self-organized $\chi^{(2)}$ grating formation $[40]$.

The mutual phase shift of the SH radiation generated and the SH seeding radiation $\Delta \varphi_{2 \omega}$ is related with the complex structure of $\chi^{(2)}$ encoding function $F(z, t-\tau)$ as well.

For purely positive imaginary $F(z, t-\tau)$ the generated $\mathrm{SH}$ radiation is in the phase with the SH seed, $\Delta \varphi_{2 \omega}=0$, and the process of $\chi^{(2)}$ grating formation is most effective when using the self-seeded self-organized $S H G, I_{2 \omega}^{\text {seed }}=0$, or a very weak SH seeding radiation, $I_{2 \omega}^{\text {seed }} / I_{\omega}^{2} \ll 10^{-16} \mathrm{~m}^{2} / \mathrm{W}$.

In the academic case, if $F(z, t-\tau)$ were purely real, the generated $\mathrm{SH}$ radiation would be shifted by $90^{\circ}$ with respect to the $\mathrm{SH}$ seeding radiation, $\Delta \varphi_{2 \omega}=$ $\pm 90^{\circ}$, and the efficiency of the preparation process should be zero. This situation really occurs in the saturation state, if $\left(I_{2 \omega} / I_{\omega}^{2}\right)_{\mathrm{sat}} \approx 10^{-16} \mathrm{~m}^{2} / \mathrm{W}$, but the absolute value of $F(z, t-\tau)$ approaches zero in this case [40].

For purely negative imaginary $F(z, t-\tau)$ there is a mutual phase shift between the $\mathrm{SH}$ seeding radiation and the $\mathrm{SH}$ radiation generated $\Delta \varphi_{2 \omega}=180^{\circ}$ there, and the $\chi^{(2)}$ grating can be written in the course of self-organized parametric down conversion (PDC) process. This can happen if $I_{2 \omega}^{\text {seed }} / I_{\omega}^{2} \approx 10^{-16} \mathrm{~m}^{2} / \mathrm{W}$.

In any case there is a tendency of self-organized $\chi^{(2)}$ grating formation to saturate, meaning that the $\chi^{(2)}$ encoding function $F(z, t-\tau)$ rotates in the complex plane towards the real axis in the course of $\chi^{(2)}$ grating formation and, simultaneously, its absolute value decreases and approaches zero in the saturation state [40].

In Anderson's et al. picture [29], if using relatively intense SH seeding being above the saturation value, $I_{2 \omega}^{\text {seed }} / I_{\omega}^{2}>\left(I_{2 \omega} / I_{\omega}^{2}\right)_{\text {sat }}$, an unstable state is created and the interaction is expected to have a tendency to saturate. This can happen due to the energy transfer from the SH radiation into the fundamental radiation, namely, the parametric down conversion. We believe that an efficient PDC can occur if the $\mathrm{SH}$ seeding radiation is relatively strong compared with the saturation value, $I_{2 \omega}^{\text {seed }} \approx 10^{-16} I_{\omega}^{(2)}$ (in SI units). The efficiency is expected to reach the value $\left[I_{\omega}^{\text {output }} /\left(I_{\omega}^{\text {input }}+I_{2 \omega}^{\text {input }}\right)\right]_{\text {sat }} \approx 1$ in the optimum case. Even when the visible green/blue light alone causes the erasure of the $\chi^{(2)}$ structure created [41], we are of the opinion that optimum pump and SH seeding light intensities can be found which are able to write very effectively stable $\chi^{(2)}$ gratings in doped glass using PDC processes.

It seems very likely that the $\chi^{(2)}$ gratings were already formed owing to the self-organized PDC in some experiments reported [27, 42, 43], if the SH seeding radiation exceeded the saturation value (see, discussion in Ref. [33]).

The first aimed experiment on self-organized PDC was performed by P. Chmela and O. Samek in the Laboratory of Nonlinear Optics, Physics Department of Pontificia Universidade Católica in Rio de Janeiro.

The experiment was carried out with the Nd:YAG laser operated Q-switched and mode-locked. The infrared radiation at $1.064 \mu \mathrm{m}$ was frequency doubled and both the infrared radiation and the $\mathrm{SH}$ green radiation at $0.532 \mu \mathrm{m}$ were launched into a germanium-doped 25 -cm long standard telecommunication optical fiber. The 
average output power of fundamental infrared radiation propagating in the fiber at the beginning of preparation process was measured to be $P_{\omega}^{\text {output }}(0)=17.85 \mathrm{~mW}$. Unfortunately, our power meter was not calibrated well for $\mathrm{SH}$ radiation so that we had to recalculate the SH power propagating in the fiber at the beginning of preparation process using the energy conservation law to be $P_{2 \omega}^{\text {output }}(0) \approx 8.5 \mathrm{~mW}$. However, not even the introduced power of fundamental infrared radiation is quite certain as well.

The relative output powers of fundamental and SH radiations measured in 5 minutes intervals are introduced in the Table.

TABLE

Relative output powers of fundamental and SH output radiations in the course of $\chi^{(2)}$ grating formation owing to the self-organized parametric down conversion.

\begin{tabular}{c|c|c}
\hline \hline Preparation time $[\mathrm{min}]$ & $P_{\omega}^{\text {output }}(t) / P_{\omega}^{\text {output }}(0)$ & $P_{2 \omega}^{\text {output }}(t) / P_{2 \omega}^{\text {output }}(0)$ \\
\hline 0 & 1 & 1 \\
5 & 1.05 & 0.84 \\
10 & 1.1 & 0.78 \\
15 & 1.1 & 0.8 \\
20 & 1.1 & 0.73 \\
25 & 1.14 & 0.7 \\
30 & 1.14 & 0.69 \\
35 & 1.15 & 0.68 \\
40 & 1.15 & 0.68
\end{tabular}

It is quite evident from the Table that the $\mathrm{SH}$ output power decreased whilst the fundamental output power increased in the course of self-organized second-order nonlinear optical interaction, which clearly demonstrates that the self-organized PDC took place in the conditioned fiber. Consequently, the SH input light intensity was above the saturation value in our experiment. Unfortunately, not all parameters were known to calculate the light intensities of interacting radiations in the fiber.

The prepared fiber was tested by launching the infrared radiation of $18.7 \mathrm{~mW}$ output power alone. The slight green radiation was generated in the prepared fiber which was about three orders weaker than we had expected. Maybe the relatively effective PDC in the course of fiber preparation process happened owing to the instantaneous self-shaped $\chi^{(2)}$ gratings which mostly disappeared after switching off the fundamental and SH radiation fields and only a residual permanent $\chi^{(2)}$ grating survived. Further experiments must be performed to elucidate this problem.

Nevertheless, the self-organized PDC was unambiquously experimentally demonstrated for the first time. 
We are of the opinion that a considerable increasing of the typical ratio $I_{2 \omega} / I_{\omega}^{2}$ above the saturation value, $\left(I_{2 \omega} / I_{\omega}^{2}\right)^{\text {seed }} \approx 10^{-16} \mathrm{~m}^{2} / \mathrm{W}$, will provide a very effective self-organized PDC that could radically reverse the further development of the research on photoinduced self-organized nonlinear optical phenomena in doped-glass waveguide structures. Many potential applications are envisioned, especially for new kinds of self-shaped amplifiers or frequency down converters.

\section{Acknowledgments}

The authors would like to thank Dr. Isabel C.S. Carvalho and Prof. Bernard Lesche for their kind assistance.

\section{References}

[1] U. Österberg, W. Margulis, Opt. Lett. 11, 516 (1986); 12, 57 (1987).

[2] R.H. Stolen, in: Nonlinear Waves in Solid State Physics, NATO ASI Series, Eds. A.D. Boardman, T. Twardowski, M. Bertollotti, Plenum Press, New York 1990 , p. 297.

[3] International Workshop on Photoinduced Self-Organized Effects in Optical Fiber, Ed. F. Ouellette, Proc. SPIE 1516, (1991).

[4] Photosensitivity and Self-Organization in Optical Fibers and Waveguides, Ed. F. Ouellette, Proc. SPIE 2044, (1993).

[5] P. Chmela, in: Modern Nonlinear Optics, Eds. M. Evans, S. Kielich, Part 1, Wiley, New York 1993, p. 249.

[6] E.M. Dianov, D.S. Starodubov, Opt. Fiber Technol. 1, 3 (1994).

[7] F.P. Payne, Electron Lett. 23, 1215 (1987).

[8] P. Chmela, Opt. Lett. 13, 669 (1988).

[9] V. Mizrahi, Y. Hibino, G.I. Stegeman, Opt. Commun. 78, 283 (1990).

[10] A. Kamal, D.A. Weinberger, W.H. Weber, Opt. Lett. 15, 613 (1990).

[11] A. Kamal, M.L. Stock, A. Szpak, C.H. Thomas, D.A. Weinberger, M. Frankel, J. Nees, K. Ozaki, J.A. Valdmanis, Technical Digest of OSA Annual Meeting, Optical Society of America, Washington (D.C.) 1990, paper PD 25.

[12] P.A. Franken, J.F. Ward, Rev. Mod. Phys. 35, 23 (1963).

[13] S. Kielich, IEEE J. Quantum Electron. QE-5, 562 (1969).

[14] J.A. Armstrong, N. Bloembergen, J. Ducuing, P.S. Pershan, Phys. Rev. 127, 1918 (1962).

[15] M.O. Okada, K. Kakizawa, S. Teiri, Opt. Commun. 18, 331 (1976); Jpn. J. Appl. Phys. 16, 55 (1977).

[16] J. Petrácek, P. Chmela, Opt. Quantum Electron. 26, 609 (1994).

[17] R.H. Stolen, H.W.K. Tom, Opt. Lett. 12, 585 (1987).

[18] B. Lesche, J. Opt. Soc. Am. B 7, 53 (1990).

[19] N.M. Lawandy, Phys. Rev. Lett. 65, 1745 (1990).

[20] N.M. Lawandy, R.L. MacDonald, J. Opt. Soc. Am. B 8, 1307 (1991). 
[21] T.E. Tsai, D.L. Griscom, SPIE Proc. 1516, 14 (1992).

[22] E.M. Dianov, P.G. Kazansky, D.Yu. Stepanov, Sov. J. Quantum Electron. 19, 575 (1989).

[23] E.M. Dianov, P.G. Kazansky, D.Yu. Stepanov, Kvant. Electron. 17, 926 (1990) (in Russian).

[24] E.M. Dianov, D.S. Starodubov, Opt. Fiber Technol. 1, 3 (1994).

[25] E.M. Dianov, P.G. Kazansky, D.S. Starodubov, D.Yu. Stepanov, Sov. Lightwave Commun. 2, 83 (1992).

[26] V. Dominic, J. Feinberg, Opt. Lett. 18, 784 (1993).

[27] D.M. Krol, D.J. Giovani, W. Pleibel, R.H. Stolen, Opt. Lett. 18, 1220 (1993).

[28] P. Chmela, J. Petráček, Opt. Commun. 156, 374 (1998).

[29] D.Z. Anderson, V. Mizrahi, J.E. Sipe, Opt. Lett. 16, 796 (1991).

[30] N.M. Lawandy, T.J. Dricscol, C.L. Adler, IEEE Proc. J. 139, 133 (1992).

[31] P. Chmela, J. Petráček, A. Romolini, T. Pascucci, R. Falciai, Opt. Commun. 113, 305 (1994).

[32] I.C.S. Carvalho, W. Margulis, B. Lesche, Opt. Lett. 16, 1487 (1991).

[33] P. Chmela, J. Petráček, A. Romolini, T. Pascucci, R. Falciai, Opt. Fiber Technol. 1, 352 (1995).

[34] M.M. Lacerda, I.C.S. Carvalho, W. Margulis, B. Lesche, Electron. Lett. 30, 732 (1994).

[35] W. Margulis, I.C.S. Carvalho, J.P. von der Weid, Opt. Lett. 14, 700 (1989).

[36] E.M. Dianov, P.G. Kazansky, D.S. Starodubov, D.Yu. Stepanov, Sov. Lightwave Commun. 1, 395 (1991).

[37] V. Dominic, J. Feinberg, SPIE Proc. 2044, 223 (1993).

[38] P. Lambelet, J. Feinberg, Opt. Lett. 21, 925 (1996).

[39] P. Chmela, J. Petráček, K. Gniadek, Opt. Quantum Electron. 30, 271 (1998).

[40] P. Chmela, J. Petráček, Opt. Commun. 156, 374 (1998).

[41] F. Ouellette, K.O. Hill, D.C. Johnson, Opt. Lett. 13, 515 (1988).

[42] G. Demouchy, G.R. Boyer, Opt. Commun. 101, 385 (1993).

[43] G. Demouchy, Opt. Commun. 101, 515 (1993). 